\title{
Klarheit für komplexe Themen
}

\section{Expertenkonsultationen mit Hilfe von Argumentationskarten}

\author{
Ralf Grötker
}

\begin{abstract}
Kernaussagen
Expertenkonsultationen ermöglichen es, in Fällen komplexer Themen und widerstreitender Meinungen $\mathrm{zu}$ einer demokratisch legitimierten Wissenssynthese zu gelangen. Durch den Einsatz von Argumentationskarten kann dieser Prozess maßgeblich unterstützt werden. Am Beispiel einer Expertendiskussion zur gesetzlichen Neuregelung von Sterbehilfe und assistiertem Suizid wird der Ablauf eines speziell für den Einsatz von Argumentationskarten konzipierten Verfahrens vorgestellt. Teilnehmer einigen sich dabei nicht auf einen Konsens oder eine gemeinsame Erklärung, sondern auf die vollständige Darstellung der Kontroverse. Dies ermöglicht komplexere Formen der Auswertung als herkömmliche Konsultationsformate.
\end{abstract}

\section{Einleitung}

Vor allem bei kontroversen und politisch aufgeladenen Fragestellungen können Expertenkonsultationen helfen, eine gleichermaßen breite wie demokratisch legitimierte Wissensbasis für politische Entscheidungen zu schaffen. Expertenkonsultationen sind zu diesem Zweck deshalb besonders geeignet, weil sie als Instrument zur Synthese von Wissen und Meinungen wichtige Defizite in der Informationslandschaft schließen:

- Begutachtungsprozesse gehen einher mit der Erzeugung großer Textvolumen. Diese entstehen durch die wiederholte Analyse, Zusammenfassung und Kompilation von Literatur. Das Resultat: wachsende Unübersichtlichkeit.

- Hinzu kommt ein Problem in der Akteurskonstellation. Von Seiten der Wissenschaft besteht insbesondere bei interdisziplinären Projekten meist für die individuellen Akteure kaum ein Anreiz, Zeit und Mühe in die Forschungssynthese zu investieren - schlicht deshalb, weil der Rahmen des gemeinsamen Projekts sehr viel weiter gesteckt ist als das eigene Forschungsfeld. Auf diese Weise kommt es dazu, dass etliche Themen, die für Öffentlichkeit und Politik von großem Interesse sind, in dem gefragten Zuschnitt von der Wissenschaft gar nicht aufgegriffen werden können.

- In Fällen, wo die politische oder mediale Öffentlichkeit involviert ist, tritt eine weitere Erschwernis hinzu. Nicht erst seit der Berichterstattung über den Klimawandel ist bekannt, dass die Auseinandersetzung mit Gegnern und Skeptikern dazu führt, dass deren Standpunkte an Glaubwürdigkeit gewinnen - selbst dann, wenn sie kritisiert oder explizit widerlegt werden. Hängen bleibt beim Rezipienten, dass es eine ernstzunehmende „Kontroverse“ gibt - selbst dann, wenn dies gar nicht der Fall ist (Brock \& Balloun, 1967; Boykoff \& Boykoff, 2004; Skurnik, 2006).

Dieser Problemlage kann mit dem Mittel der Expertenkonsultation erfolgreich begegnet werden:

- Moderation und Strukturierung der Prozesse dämmen die Informationsüberflutung ein.

- Der Veranstalter eines Konsultationsprozesses nimmt die Rolle eines Mittlers ein, der die Zusammenführung von Expertise als seine eigene Aufgabe begreift.

n Im geschützten Rahmen fortlaufender gemeinsamer Überlegungen lassen sich die beschriebenen psychologischen Fallen vermeiden.

Allerdings muss der Konsultationsprozess so gestaltet werden, dass die möglichen Potenziale auch realisiert werden können. Dieses Ziel verfolgt das Verfahren von Expertenkonsultationen mit Hilfe von visualisierter Problemstrukturierung für Planen und Entscheiden im Team, FürundWider. Das Verfahren wurde im Kontext der Plattform Debattenprofis.de entwickelt und in den vergangenen Jahren in mehreren Pilotprojekten getestet.

\section{Das Verfahren}

Ähnlich wie beim klassischen Delphi Verfahren führt bei der Konsultation mit Hilfe von visualisierter Problemstrukturierung FürundWider ein Team von Moderatoren wiederholte Einzelbefragungen von Mitgliedern eines Expertenteams durch. Anderes als beim Delphi Verfahren ist das Ziel eines FürundWider jedoch nicht die Abfrage von subjektiven Schätzwerten für Daten und Fakten, sondern vielmehr die Darstellung eines argumentativen Raumes. Die Interviews dienen dazu, ein detailliertes Bild des Für und Wider bezüglich einer Streitfrage abzubilden. Mit diesem Gesamtbild, nicht jedoch mit der Bewertung der einzelnen Positionen, sollen am Ende des Prozesses alle Teilnehmer einverstanden sein.

Das Verfahren wird maßgeblich unterstützt durch den Einsatz von Argumentationskarten. Vom Ansatz her ähneln Argumentationskarten Mindmaps: Beides sind grafische Darstellungen von Textelementen. Grafische Darstellungen dieser Art haben Vorteile gegenüber rein textlichen Präsentationen: Die baum- oder netzwerkartige Struktur erlaubt es, einfacher zwischen den verschiedenen Ebenen oder Zweigen zu navigieren und einzelne Punkte zu fokussieren. Anders als Mindmaps sind Argumentationskarten keine Darstellung von Themen und Unterthemen. Stattdessen stellen sie eine Beweiskette dar - eine idealisierte Beweiskette. Sinn und Zweck einer Argumentationskarte ist nicht, tatsächliche 'unordentlichen' Prak- 
tiken durch eine Art Kalkül zu ersetzen, sondern vielmehr, den fortlaufenden Überlegungsprozess zu unterstützen.

Am Ende einer Beweiskette findet sich eine „Basis“. Basiselemente geben eine Antwort auf die Frage „Woher will man das wissen? “. Die Basis gibt Auskunft darüber, welche Art von Evidenzen eine gegebene Behauptung belegen - wie etwa statistische Daten, anekdotische Beispiele oder ein Laborexperiment. Ein Blick auf die Karte zeigt so, welche Behauptungen durch eine Basis gedeckt werden und welche nicht. Oft ist die Basis ein guter Ansatzpunkt, um eine Behauptung in Frage zu stellen: „Belegt die angeführte Evidenz wirklich die betreffende Behauptung? “

Weitere Vorteile von Argumentationskarten zur Unterstützung von kollaborativem Planen und Entscheiden und sind:

- Klarheit darüber, welche Themen bereits in die Diskussion eingebracht und wie ausführlich diese besprochen wurden.

- Wer etwas ergänzen will, kann dies tun, ohne sich zuvor durch dutzende Seiten von Vorgänger-Kommentaren zu arbeiten und ohne bereits Gesagtes wiederholen zu müssen.

- Versachlichung: ,nackte'Argumente treten an die Stelle von Reputation und Rhetorik

Der Einsatz von Argumentationskarten für Planen und Entscheiden im Team ist in den vergangenen Jahren im Rahmen verschiedenster Szenarios erfolgreich getestet worden (Berardi, Bachler, Bernhard, Shum, Ganapathy, Mistry, Reynolds \& Ulrich, 2006; Conklin, 2005; Culmsee and Awatai, 2011; Klein 2007, Okada, Shum \& Sherbone, 2008; Scheuer, Pinkwart \& McLaren, 2010, Selvin, 2011). Auch zur Moderation von Stakeholder-Dialogen ist die Technik bereits Ende der 1990er Jahre eingesetzt worden (van den Daele, Pühler \& Sukopp, 1996): Damals ging es um eine partizipative Technikfolgenabschätzung zu gentechnisch auf Schädlingsresistenz hin optimierter Nutzpflanzen. Erst die in allerjüngster Zeit erfolgte Verbreitung einer Reihe von Anwendungen für online-unterstützte Teamarbeit macht es jedoch möglich, ohne aufwändige technische Ausstattung auch über räumliche Distanzen hinweg mit Argumentationskarten zu arbeiten. $\mathrm{Zu}$ diesen Anwendungen zählen:

- File-Sharing Dienste (wie die „Dropbox“)

- Screen Sharing (zum Beispiel via Skype)

- Funktionen, mit denen sich Dokumente und auch Grafiken im Team bearbeiten und kommentieren lassen (seit kurzem erst beinhaltet der kostenlose Adobe PDF-Reader auch eine Kommentierungsfunktion)

- Online-Meinungsumfragen innerhalb von geschlossenen Gruppen (etwa mit Google Spreadsheets)

Hinzu kommen speziell für den Online-Einsatz entwickelte, zum Teil jedoch noch im Entwicklungsstadium befindliche Anwendungen wie zum Beispiel Agora (agora.gatech.edu), Argunet, Debategraph, B-Cisive Online oder die im Entstehen begriffene Toolbox des EU-Forschungsprojekts Integrated Method for Policy Making Using Argument Modelling and
Computer Assisted Text Analysis (Impact). Von den speziellen Anwendungen abgesehen wird die Handhabung großformatiger Diagramme, wie Argumentationskarten sie oft darstellen, erleichtert durch den zunehmenden Einsatz von Smart Boards. Mit der neueren Version des (kostenlosen) Adobe Acrobate Readers ist zudem der Ausdruck großformatiger Diagramme über mehrere DIN A 4-Seiten verteilt auf einfache Weise möglich.

Um auszutesten, wie in einen Online-Auftritt eingebettete Argumentationskarten Expertenkonsultationen unterstützen können, wurde vom Verfasser dieses Beitrags zusammen mit dem Journalistenbüro „Schnittstelle“ die Plattform Debattenprofis.de (vormals FürundWider.org) ins Leben gerufen. Neben Argumentationskarten beinhaltet die Plattform Einführungstexte, Kommentare und Zusammenfassungen des Diskussionsstandes zu dem jeweiligen Thema. Ein Moderator hat die Aufgabe, Teilnehmer eines FürundWider gezielt auf offene Fragen anzusprechen und eingehende Beiträge in die Debattenkarten einzubauen. Thematisch konzentriert sich die Plattform auf aktuelle gesellschaftliche Fragen mit wissenschaftlichem Hintergrund. Das Verfahren wurde erstmals vorgestellt auf dem Media for Science Forum 2010 in Madrid und dem Workshop Emerging Tools im Rahmen der Online Deliberation 2010 in Leeds. Die Plattform wird unterstützt unter anderem von der Max Planck Gesellschaft, der Leibniz Gemeinschaft und der Wissenschafts-Pressekonferenz e.V.

Im November 2010 wurde als Pilotprojekt ein Fürund Wider zum Thema "Synthetische Biologie“ durchgeführt. Das Projekt wurde von der Deutschen Akademie der Technikwissenschaften acatech, die als „Debattenpate“ fungierte, finanziell unterstützt. Ein weiteres Projekt im Frühjahr 2011 befasste sich mit der Diskussion um eine gesetzliche Neuregelung von Sterbehilfe und assistiertem Suizid in Deutschland. Debattenpate war die Heinrich Böll Stiftung. Die redaktionelle Unabhängigkeit der Plattform blieb in beiden Projekten gewahrt.

\section{Durchführung}

$\mathrm{Zu}$ Beginn eines FürundWider stehen eine umfangreiche Recherche und eine erste Problemstrukturierung. Dabei kommen so genannte Argumentationsschemata zum Einsatz: Schablonen, die bei der Zergliederung von komplexen Themen helfen und, wichtiger noch, auf systematischen Weg zu einer Reihe von kritischen Fragen oder Einwänden führen, die es zu prüfen gilt (Atkinson, Bench-Capon \& McBurney, 2006).

Teil der Basisrecherche ist die Erstellung einer Liste geeigneter Teilnehmer. Im Fall des FürundWider zum Thema Sterbehilfe waren dies Mediziner, Juristen, Ethiker, Soziologen sowie Vertreter von Bürgerrechtsvereinigungen und Anbieter von Beratungsdienstleistungen bezüglich von Patientenverfügungen. Ziel der Teilnehmerakquise war es, eine Gruppe von insgesamt 15 bis 20 Experten zu gewinnen. Um dieses Ziel zu erreichen, wurden insgesamt 25 Experten angefragt. Eine Gruppe bestehend aus dem ärztlichen Leiter eines Unfallkrankenhauses, einem Palliativmediziner, einem Strafrechtler, 
einem Verfassungsrechtler, einem Gesundheitssoziologen und einer Philosophin wurde als Jury gewonnen. Hauptaufgabe der Jury war es, am Ende des Fürund Wider die Vollständigkeit der abgebildeten Argumente zu beurteilen. Darüber hinaus standen die Jurymitglieder auch für eine abschließende Befragung zur Verfügung. Weitere zehn Teilnehmer wurden als Mitglieder des Expertenteams rekrutiert.

Die Interviews mit Jury und Experten fanden telefonisch oder bei persönlichen Treffen statt und dauerten meist ein bis zwei Stunden. Die Gesprächsführung orientierte sich nicht an einem einheitlichen Fragenkatalog, sondern folgte dem sich über den Projektzeitraum weiter entwickelnden Stand der Recherche. Ergebnisse der Interviews wurden protokolliert, sich daraus ergebende Änderungen im Diskussionsstand zusammengefasst und auf der Webseite veröffentlicht.

Parallel zu den sich über einen Zeitraum von mehreren Wochen erstreckenden Konsultationen von Jury und Expertenteam wurde die auf Grundlage der Ausgangsrecherche erstellte Problemstrukturierung zu einer detaillierten Argumentationskarte ausgebaut (siehe: www.sterbehilfe.fuerundwider.org). Die jeweils aktuelle Version der Karte wurde ebenfalls über die Webseite veröffentlicht. Die Karte wurde außerdem von der Moderation zur Strukturierung der Interviews verwendet. Eine Befragung der Jurymitglieder bildete den Schlusspunkt der Konsultationsphase.

Die Auswertung des FürundWider erfolgte in Form eines Abschlussberichtes (Grötker \& Linhart, 2012). Bei der Erstellung des Berichts wurde Wert darauf gelegt, dass dieser auch solchen Adressaten zugänglich sein sollte, die mit dem visuellen Format der Argumentationskarten nicht vertraut sind. Erste Ergebnisse wurden im Vorfeld bereits auf der Veranstaltung „Die Freiheit zu sterben. Nachdenken über assistierten Suizid und aktive Sterbehilfe “ präsentiert, welche die Heinrich Böll Stiftung zusammen mit der Humanistischen Union und der Deutschen Gesellschaft für Humanes Sterben am 14. April 2011 in Berlin organisierte.

\section{Ergebnisse}

Die Argumentationskarten erwiesen sich insgesamt als hilfreiches Instrument zum Zwecke des persönlichen Wissensmanagement. Ohne die Karten hätte die Moderation die Komplexität des Sachverhaltes kaum in der Kürze der gegebenen Zeit angemessen durchdringen können. Aus Jury und Expertenteam nutzen allerdings nur wenige Teilnehmer die Karten - einige davon jedoch intensiv. Abgesehen von der unmittelbaren Verwendung spielten die Argumentationskarten für den gesamten Prozess insofern eine wichtige Rolle, als sie dem Fürund Wider ein ,Gesicht' verliehen und auch eine klare Zielsetzung definierten. Diese bestand in einer Zusammenschau der in die Diskussion eingebrachten Argumente, wie es sie bis dato noch nicht gab. Für viele Teilnehmer war dies ein wichtiger Anreiz dafür, sich zu beteiligen.

In inhaltlicher Hinsicht überraschte, dass die großen Meinungsverschiedenheiten nicht entlang der Linie ethischer
Grundsatzdifferenzen verliefen, so wie dies zum Thema Sterbehilfe vielleicht zu erwarten gewesen wäre. Im Vordergrund standen stattdessen unterschiedliche Auffassungen darüber, was gesetzliche Regulierung oder Klarstellung bewirkt und wie wünschenswert diese Effekte sind. Dabei ging es zum einem um Sorgen hinsichtlich negativer Folgen einer „Verrechtlichung“, zum anderen um Befürchtungen einer „Normerosion“ durch eine freizügigere Gesetzgebung.

Im Gesamtbild fiel vor allem auf, dass die Meinungen keinem erkennbaren Grobmuster folgten. Stattdessen ließ sich eine Vielzahl unterschiedlicher Argumentationsfiguren ausmachen. Von Zustimmung oder Ablehnung einzelner Argumentationspunkte kann somit nicht auf die Akzeptanz anderer Punkte geschlossen werden.

\section{Diskussion}

Das durch die Expertenkonsultation mit Hilfe von Argumentationskarten erzeugte Meinungsbild macht deutlich, inwiefern ein zusammenfassendes Urteil abhängig ist von der Wahl des Auswertungsverfahrens. Bildet man zum Beispiel den Durchschnitt der Expertenmeinungen, dann gleichen sich Ablehnung und Akzeptanz einzelner Punkte gegenseitig aus. Das Ergebnis ist ein Meinungsbild, das im beschriebenen Fall nicht der Argumentationslinie auch nur eines einzigen der befragten Experten entspricht. Dies deutet darauf hin, dass es im gegebenen Fall, aber auch in anderen Situationen sinnvoll sein könnte, Annäherungsbereitschaften zwischen entgegengesetzten Positionen anders als durch Durchschnittsbildung auszuloten. Hierbei gilt es zwischen verschiedenen Zielsetzungen zu unterscheiden. Unter heuristischen Gesichtspunkten würde es sich anbieten, Einzelaspekte herauszugreifen wie etwa die Prognose von Folgen gesetzlicher Regulierung. Hier könnte es sehr wohl hilfreich sein, Expertenmeinungen durch Verfahren der Durchschnittsbildung zu aggregieren. Was die politischen Leitideen betrifft, könnte es weiter helfen, die Wünschbarkeit von Zielen wie Transparenz („Sterbehilfe beim Namen nennen“) auf den Prüfstand zu stellen. Noch einmal anders liegen die Dinge, wenn eine Konfliktmediation gefragt ist. Die Argumentationskarten und Protokolle enthalten zahlreiche Hinweise darauf, wie eine Annäherung der beteiligten Stakeholder geschehen könnte - insofern Karten und Protokolle nämlich nicht nur widerstreitende Meinungen darstellen, sondern auch die Gründe offen legen, weshalb Teilnehmer Punkte in der Diskussion unterschiedlich bewerten. Die Suche nach gemeinsamen Nennern könnte bei diesen Gründen ansetzen.

Es bleibt die Frage, unter welchen Umständen sich ein FürundWider erfolgreich einsetzen lässt. Eine Grundvoraussetzung für das Gelingen dieser Art von „Crowdsourcing“ scheint die Arbeit mit relativ überschaubaren Teams zu sein, die persönlich angesprochen werden. Eine weitere Erfolgsbedingung ist, dass das Ergebnis einer Konsultation praktische Relevanz haben muss. Im beschriebenen Fall war dies durch die Präsenz auf der Veranstaltung „Die Freiheit zu sterben“ 
gegeben sowie durch die Publikation des Abschlussberichts im Rahmen der Veranstaltungsdokumentation.

Schließlich scheinen nicht alle Fragestellungen gleichermaBen für Expertenkonsultationen nach dem Modell eines FürundWider geeignet zu sein. Aus unserer eigenen Erfahrungen und aus der Beschäftigung mit Projekten aus dem Umfeld von Open Government stellen sich folgende Faktoren als relevant für die Erfolgschancen von "offenen Expertisen“ nach Art eines FürundWider dar (vgl. auch Schaal \& Ritzi, 2009):

- Die Teilnehmer verfügen über ein Wissen, das nur sie beisteuern können.

- Teilnehmer haben ein Eigeninteresse entweder am Ausgang einer Debatte oder an der Präsenz einer Debatte.

- Konflikte stellen sich nicht von vornherein als unüberbrückbar dar.

- Meinungsunterschiede verlaufen nicht ausschließlich entlang von vornherein leicht absehbaren Interessenkonflikten.

- Ein praktisches Ziel ist in Sicht.

Diese Bedingungen waren im Fall der Diskussion um die Sterbehilfe in hervorragendem Maße erfüllt.

\section{Literatur}

Atkinson, K., Bench-Capon, T., \& McBurney, P. (2006). Computational Representation of Practical Argument. Synthese, 22(2), 157-206.

Berardi, A., Bachler, M., Bernard, C., Shum, S.B., Ganapathy, S., Mistry, J., Reynolds, M. \& W. Ulrich (2006). The ECOSENSUS Project: CoEvolving Tools, Practices and Open Content for Participatory Natural Resource Management. In: Second International Conference on e-Social Science, 28-30 June 2006, Manchester.

Boykoff, M. T., \& Boykoff, J. M. (2004). Balance as bias: global warming and the US prestige press. Global Environmental Change 14(2), 125-136. Brock, T. C., \& Balloun, J. L. (1967). Behavioral receptivity to dissonant information. Journal of Personality and Social Psychology, 6(4, Pt.1), 413-428.

Conklin, J. (2005). Dialogue Mapping: Building Shared Understanding of Wicked Problems. Wiley.

Culmsee, P. \& Awati, K. (2011). The Heretic's Guide to Best Practices: The "Reality " of Managing Complex Problems in Organisations. iuniverse.com.

Daele, W. van den, Pühler, A. \& Sukopp, H. (1996). Transgenic Herbidcide-Resistant Crops: A Participatory Technology Assessment. Summary Report. WZB Discussion Paper FS II 97-302.

Grötker, R., \& Linhart, J. (2012). Die Webseite sterbehilfe.fuerundwider.org. In Heinrich Böll Stiftung (Hg.), Selbstbestimmung am Lebensende. Nachdenken über assistierten Suizid und aktive Sterbehilfe. (S. 120-146). Berlin: Heinrich Böll Stiftung. http://www.sterbehilfe.fuerundwider.org/ abschluss_sterbehilfe.pdf [

Klein, M. (2007). The MIT Collaboratorium: Enabling Effective LargeScale Deliberation for Complex Problems. SSRN eLibrary. http:// papers.ssrn.com/sol3/papers.cfm?abstract_id=1085295.

Okada, A., Shum, S.J.B., \& Sherborne, T. (2008). Knowledge Cartography: Software Tools and Mapping Techniques. Springer-Verlag.

Schaal, G. S. \& Ritzi, C. (2009). Empirische Deliberationsforschung. MPIfG Working Paper 09/09. www.mpifg.de/pu/workpap/wp09-9.pdf.

Scheuer, O., Loll,F. Pinkwart, N. \& B. M. McLaren. (2010). Computersupported argumentation: A review of the state of the art. International Journal of Computer-Supported Collaborative Learning 5(1), 43-102.

Selvin, A. M. (2011) Making Representations Matter: Understanding Practitioner Experience in Participatory Sensemaking. Doctoral Dissertation. Technical Report KMI-11-03. Knowledge Media Institute, The Open University,

Skurnik, I. (2006). Memory for flu facts and myths and effects on vaccine intentions. ClinicalTrials.gov identifier. http://clinicaltrials.gov/ct $2 /$ show/ NCT00296270.

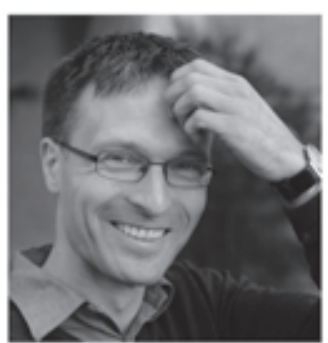

Foto: David Ausserhofer
Ralf Grötker ist Gründer der Plattform Debattenprofis.de. Er studierte Philosophie und Kulturwissenschaften in Bremen, Paris und Köln und promovierte an der FU Berlin. Er ist freier Wissenschaftsjournalist und war dazu u.a. „Journalist in Residence $^{*}$ am MPIfG und am WZB sowie Fellow am IGK „Arbeit und Lebenslauf" der HU Berlin. E-Mail: groetker@explorat.de.

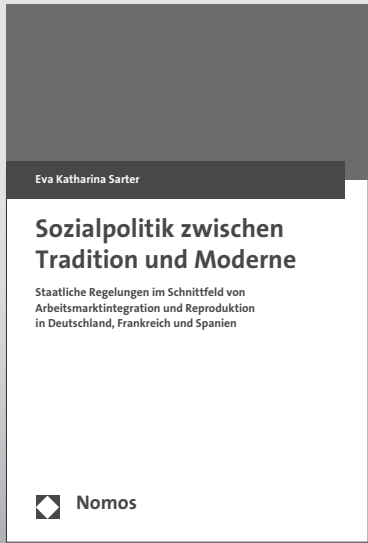

\section{Sozialpolitik zwischen Tradition und Moderne}

Staatliche Regelungen im Schnittfeld von Arbeitsmarktintegration und Reproduktion in Deutschland, Frankreich und Spanien

Von Eva Katharina Sarter

2012, ca. 240 S., brosch., ca. 44,- $€$

ISBN 978-3-8329-7601-9

Erscheint ca. September 2012 